\title{
The Stability of a General Sextic Functional Equation by Fixed Point Theory
}

\author{
Jaiok Roh $\mathbb{D}^{1}{ }^{1}$ Yang-Hi Lee, ${ }^{2}$ and Soon-Mo Jung $\mathbb{D}^{3}$ \\ ${ }^{1}$ Ilsong College of Liberal Arts, Hallym University, 200-702 Chuncheon, Republic of Korea \\ ${ }^{2}$ Department of Mathematics Education, Gongju National University of Education, Gongju 32553, Republic of Korea \\ ${ }^{3}$ Mathematics Section, College of Science and Technology, Hongik University, 30016 Sejong, Republic of Korea
}

Correspondence should be addressed to Jaiok Roh; joroh@hallym.ac.kr

Received 28 February 2020; Revised 22 September 2020; Accepted 6 October 2020; Published 24 December 2020

Academic Editor: Raúl E. Curto

Copyright ( 92020 Jaiok Roh et al. This is an open access article distributed under the Creative Commons Attribution License, which permits unrestricted use, distribution, and reproduction in any medium, provided the original work is properly cited.

In this paper, we will consider the generalized sextic functional equation $\sum_{i=0}^{7} C_{i}(-1)^{7-i} f(x+i y)=0$. And by applying the fixed point theorem in the sense of Cadariu and Radu, we will discuss the stability of the solutions for this functional equation.

\section{Introduction}

In 1940, Ulam [1] remarked the problem concerning the stability of group homomorphisms. In 1941, Hyers [2] gave an answer to this question for additive mappings between Banach spaces. Subsequently, many mathematicians came to deal with this question (cf. [3-10]). Let $V$ and $W$ be real vector spaces, $X$ be a real normed space, $Y$ be a real Banach space, $n \in \mathbb{N}$ (the set of natural numbers), and $f: V \longrightarrow W$ be a given mapping. Consider the functional equation

$$
\sum_{i=0}^{n}{ }_{n} C_{i}(-1)^{n-i} f(i x+y)-n ! f(x)=0
$$

for every $x, y \in V$, where ${ }_{n} C_{i}=n ! / i !(n-i) !$. The functional equation (1) is called an $n$-monomial functional equation, and every solution of the functional equation (1) is called to be a monomial mapping of degree $n$. The function $f: \mathbb{R} \longrightarrow \mathbb{R}$ given by

$$
f(x)=a x^{n},
$$

is a particular solution of the functional equation (1). In particular, the functional equation (1) is called a sextic functional equation for the case $n=6$, and every solution of the functional equation (1) is called to be a sextic mapping for the case $n=6$. Many mathematicians [11-17] have previously investigated the stability of the sextic functional equation, and many authors [18-26] have studied the stability of the $n$-monomial functional equation in various spaces.

The solution of the functional equation

$$
\sum_{i=0}^{n}{ }_{n} C_{i}(-1)^{n-i} f(x+i y)=0,
$$

is called a generalized polynomial mapping of degree $n$ $\in \mathbb{N}$ (See Baker [27]). The function $f: \mathbb{R} \longrightarrow \mathbb{R}$ given by

$$
f(x)=\sum_{i=0}^{n} a_{i} x^{i}
$$

is a particular solution of the functional equation (3). Some mathematicians [28-31] have previously investigated the stability of the functional equation (3) for the cases $n=4,5,6,7$. In particular, the functional equation

$$
\sum_{i=0}^{7}{ }_{7} C_{i}(-1)^{7-i} f(x+i y)=0,
$$

is called a general sextic functional equation, and every solution of the functional equation (4) is said to be the general sextic mapping. 
In this paper, we will partially generalize the results in [31] for the stability of the general sextic functional equation. For the details, one can refer Corollary 4 and Corollary 7 which are special cases of main theorems. Specifically, in this paper, we will show that there is only one solution $F$ of the general sextic functional equation (4) near the function $f$, which approximates the functional equation (4) by using fixed point theorem [32-35]. Moreover, the solution mapping $F$ of the functional equation (4) can be explicitly constructed by the formula

$$
\begin{aligned}
F(x)=\lim _{n \rightarrow \infty} \sum_{i=0}^{n}{ }_{n} C_{i} \sum_{j=0}^{i}{ }_{i} C_{j}\left(\frac{(-84)^{n-i} 1344^{i}}{4096^{n}} f_{e}\left(2^{2^{3 n-i-j}} x\right)\right. \\
\left.+\frac{(-42)^{i-j} 336^{j}}{512^{n}} f_{o}\left(2^{3 n-i-j} x\right)\right)
\end{aligned}
$$

or

$$
\begin{aligned}
F(x)=\lim _{n \rightarrow \infty} & \sum_{i=0}^{n}{ }_{n} C_{i} \sum_{j=0}^{i}{ }_{i} C_{j}\left(42^{j}(-336)^{i-j} 512^{n-i} f_{o}\left(\frac{x}{2^{3 n-i-j}}\right)\right. \\
& \left.+84^{j}(-1344)^{i-j} 4096^{n-i} f_{e}\left(\frac{x}{2^{3 n-i-j}}\right)\right),
\end{aligned}
$$

which approximates the mapping $f$.

\section{Main Results}

We first recall the following Margolis and Diaz fixed point theorem, which is necessary to obtain the main results of this paper.

Proposition 1 (see [36]). Suppose $(X, d)$ is a complete generalized metric space, which means that the metric $d$ may assume infinite values, and $J: X \longrightarrow X$ is a strictly contractive mapping with the Lipschitz constant $0<L<1$. Then, for each given element $x \in X$, either

$$
d\left(J^{n} x, J^{n+1} x\right)=+\infty \text { for all } n \in \mathbb{N} \cup\{0\}
$$

or there exists an integer $k \geq 0$ such that:

(i) $d\left(J^{n} x, J^{n+1} x\right)<+\infty$ for all $n \geq k$

(ii) The sequence $\left\{J^{n} x\right\}$ converge to a fixed point $y^{*}$ of $J$

(iii) $y^{*}$ is a unique fixed point of $J$ in $Y=\left\{y \in X: d\left(J^{k}\right.\right.$ $x, y)<+\infty\}$

(iv) $d\left(y, y^{*}\right) \leq 1 /(1-L) d(y, J y)$ for every $y \in Y$

In this paper, we let $V$ and $W$ be real vector spaces, $X$ be a real normed space, and $Y$ be a real Banach space. For a mapping $g: V \longrightarrow W$, we use the following abbreviations

$$
\begin{aligned}
g_{e}(x) & =\frac{g(x)+g(-x)}{2}, \\
g_{0}(x) & =\frac{g(x)-g(-x)}{2}, \\
D g(x, y) & =\sum_{i=0}^{7}{ }_{7} C_{i}(-1)^{7-i} g(x+i y),
\end{aligned}
$$

for every $x, y \in V$.

Now, we will see useful lemma for the proof of main theorem.

Lemma 2. Let $\theta$ be a real constant such that $0<\theta<\pi / 4$ and $\cos (3 \theta)=-17 /(21 \sqrt{21})$. Let $\varphi: V^{2} \longrightarrow[0, \infty)$ be a function for which there exists a constant $0<L<1$ such that

$$
\varphi(2 x, 2 y) \leq(4 \sqrt{21} \cos \theta-14) L \varphi(x, y)
$$

for all $x, y \in V$. Then, $1<4 \sqrt{21} \cos \theta-14<2$,

$\frac{(4 \sqrt{21} \cos \theta-14)^{3}+42(4 \sqrt{21} \cos \theta-14)^{2}+336(4 \sqrt{21} \cos \theta-14)}{512}=1$,

and the equality

$\lim _{n \rightarrow \infty} \frac{1}{512^{n}} \sum_{i=0}^{n}{ }_{n} C_{i}\left(\sum_{j=0}^{i}{ }_{i} C_{j} 42^{i-j} 336^{j} \varphi_{e}\left(2^{3 n-i-j} x, 2^{3 n-i-j} y\right)\right)=0$,

holds for all $x, y \in V$.

Proof. When $0<\theta<\pi / 4$ and $\cos 3 \theta=-17 /(21 \sqrt{21})$, it is not difficult to see that $1.74837<3 \theta<1.7484$ and $0.83493<\cos$ $\theta<0.834925$ in the trigonometric function table. So $1.3<4$ $\sqrt{21} \cos \theta-14<1.4$.

We can also obtain the equality (7) by the following calculation:

$$
\begin{aligned}
& \frac{336(4 \sqrt{21} \cos \theta-14)}{512}+\frac{42(4 \sqrt{21} \cos \theta-14)^{2}}{512}+\frac{(4 \sqrt{21} \cos \theta-14)^{3}}{512} \\
& =\frac{1344 \sqrt{21} \cos \theta-4704}{512}+\frac{14112 \cos ^{2} \theta-4704 \sqrt{21} \cos \theta+8232}{512} \\
& =\frac{336 \sqrt{21}\left(4 \cos ^{3} \theta-3 \cos \theta\right)+784}{512} \\
& =\frac{336 \sqrt{21} \cos 3 \theta+784}{512} \\
& =\frac{336 \sqrt{21} \times(-17 /(21 \sqrt{21}))+784}{512}=1 .
\end{aligned}
$$


And, to obtain the equality (8), by (7), we obtain the following calculation:

$$
\begin{aligned}
\lim _{n \rightarrow \infty} & \frac{1}{512^{n}} \sum_{i=0}^{n}{ }_{n} \mathrm{C}_{i}\left(\sum_{j=0}^{i}{ }_{i} \mathrm{C}_{j} 42^{i-j} 336^{j} \varphi_{e}\left(2^{3 n-i-j} x, 2^{3 n-i-j} y\right)\right) \\
& \leq \lim _{n \rightarrow \infty} \frac{1}{512^{n}} \sum_{i=0}^{n}{ }_{n} \mathrm{C}_{i}\left(\sum_{j=0}^{i}{ }_{i} \mathrm{C}_{j} 42^{i-j} 336^{j}(4 \sqrt{21} \cos \theta-14)^{2 n-i-j} L^{2 n-i-j} \varphi_{e}\left(2^{n} x, 2^{n} y\right)\right) \\
& \leq \lim _{n \rightarrow \infty} \frac{1}{512^{n}} \sum_{i=0}^{n}{ }_{n} \mathrm{C}_{i}(4 \sqrt{21} \cos \theta-14)^{2 n-2 i}\left(\sum_{j=0}^{i}{ }_{i} \mathrm{C}_{j} 42^{i-j}(4 \sqrt{21} \cos \theta-14)^{i-j} 336^{j}\right) \times \varphi_{e}\left(2^{n} x, 2^{n} y\right) \\
& =\lim _{n \rightarrow \infty} \frac{1}{512^{n}} \sum_{i=0}^{n}{ }_{n} \mathrm{C}_{i}(4 \sqrt{21} \cos \theta-14)^{2 n-2 i}(42(4 \sqrt{21} \cos \theta-14)+336)^{i} \varphi_{e}\left(2^{n} x, 2^{n} y\right) \\
& \leq \lim _{n \rightarrow \infty} \frac{1}{512^{n}}\left((4 \sqrt{21} \cos \theta-14)^{2}+42(4 \sqrt{21} \cos \theta-14)+336\right)^{n} \varphi_{e}\left(2^{n} x, 2^{n} y\right) \\
& =\lim _{n \rightarrow \infty} L^{n}\left(\frac{(4 \sqrt{21} \cos \theta-14)^{3}+42(4 \sqrt{21} \cos \theta-14)^{2}+336(4 \sqrt{21} \cos \theta-14)}{512} \times \varphi_{e}(x, y)\right. \\
& =\lim _{n \rightarrow \infty} L^{n} \varphi_{e}(x, y)=0, \quad \text { for all } x, y \in V .
\end{aligned}
$$

In the following main theorem, we will prove the generalized Hyers-Ulam stability of the functional equation (4) by using the direct method.

Theorem 3. Let $\theta, L$, and $\varphi$ be as in Lemma 2. If $: V \longrightarrow Y$ is a mapping satisfying $f(0)=0$ and the inequality

$$
\|D f(x, y)\| \leq \varphi(x, y), \quad \text { for all } \quad x, y \in V,
$$

then there exists the unique solution mapping $F: V \longrightarrow Y$ of (4) such that

$$
\|f(x)-F(x)\| \leq \frac{\Phi(x)}{1-L}
$$

for all $x \in V$, where

$\Phi(x)=\frac{9 \varphi_{e}(-6 x, 2 x)+56 \varphi_{e}(-x, x)+392 \varphi_{e}(-2 x, x)+1008 \varphi_{e}(-3 x, x)}{4096}$.

In particular, $F$ is represented by

$$
\begin{aligned}
F(x)= & \lim _{n \rightarrow \infty} \sum_{i=0}^{n} \sum_{j=0}^{i}{ }_{n} C_{i i} C_{j}\left(\frac{(-84)^{i-j} 1344^{j}}{4096^{n}} f_{e}\left(2^{3 n-i-j} x\right)\right. \\
& \left.+\frac{(-42)^{i-j} 336^{j}}{512^{n}} f_{o}\left(2^{3 n-i-j} x\right)\right),
\end{aligned}
$$

for all $x \in V$.
Proof. We let the set $S$ be the set of the functions $g: V \longrightarrow Y$ with $g(0)=0$. And we define a generalized metric on Sby

$$
d(g, h)=\inf \left\{K \in R_{+} \mid\|g(x)-h(x)\| \leq K \Phi(x) \text { for all } x \in V\right\} .
$$

Then, it is not so difficult to show that $(S, d)$ is a complete generalized metric space (see ([34], Theorem 2.5) or the proof of ([37], Theorem 3.1)). Next, we see the mapping $J: S \longrightarrow S$, which is defined by

$$
\begin{aligned}
J g(x)= & \frac{4032 g(2 x)}{8192}-\frac{1344 g(-2 x)}{8192}-\frac{420 g(4 x)}{8192} \\
& +\frac{252 g(-4 x)}{8192}+\frac{9 g(8 x)}{8192}-\frac{7 g(-8 x)}{8192}
\end{aligned}
$$

for all $x \in V$.

And, by using the oddness and the evenness of $g_{o}$ and $g_{e}$ and ${ }_{n} C_{i-1}+{ }_{n} C_{i}={ }_{n+1} C_{i}$, due to mathematical induction, we can get

$$
\begin{aligned}
J^{n} g(x)= & \sum_{i=0}^{n}{ }_{n} C_{i} \sum_{j=0}^{i}{ }_{i} C_{j}\left(\frac{(-84)^{i-j} 1344^{j}}{4096^{n}} g_{e}\left(2^{3 n-i-j} x\right)\right. \\
& \left.+\frac{(-42)^{i-j} 336^{j}}{512^{n}} g_{o}\left(2^{3 n-i-j} x\right)\right)
\end{aligned}
$$

holds for all $n \in \mathbb{N}$ and $x \in V$.

Let $g, h \in S$ and we choose $K \in[0, \infty]$ as an arbitrary constant with $d(g, h) \leq K$. Due to the definition of $d$ and (7) in 
Lemma 2, we have

$$
\begin{aligned}
\| & J g(x)-J h(x) \| \\
\leq & \frac{4032}{8192}\|g(2 x)-h(2 x)\|+\frac{1344}{8192}\|g(-2 x)-h(-2 x)\| \\
& +\frac{420}{8192}\|g(4 x)-h(4 x)\|+\frac{252}{8192}\|g(-4 x)-h(-4 x)\| \\
& +\frac{9}{8192}\|g(8 x)-h(8 x)\|+\frac{7}{8192}\|g(-8 x)-h(-8 x)\| \\
\leq & K\left(\frac{336}{512} \Phi(2 x)+\frac{42}{512} \Phi(4 x)+\frac{1}{512} \Phi(8 x)\right) \\
\leq & K\left(\frac{336(4 \sqrt{21} \cos \theta-14) L}{512}+\frac{42(4 \sqrt{21} \cos \theta-14)^{2} L^{2}}{512}\right. \\
& \left.+\frac{(4 \sqrt{21} \cos \theta-14)^{3} L^{3}}{512}\right) \Phi(x) \\
\leq & K\left(\frac{(336(4 \sqrt{21} \cos \theta-14)}{512}+\frac{42(4 \sqrt{21} \cos \theta-14)^{2}}{512}\right. \\
& \left.+\frac{(4 \sqrt{21} \cos \theta-14)^{3}}{512}\right) L(x) \leq L K \Phi(x),
\end{aligned}
$$

for every $x \in V$, which implies that

$$
d(J g, J h) \leq L d(g, h)
$$

for all $g, h \in S$, where $0<L<1$. That is, with the Lipschitz constant $L, J$ is a strictly contractive self-mapping of $S$, where $0<L<1$.

Now, after long and tedious calculation, we have

$$
\begin{aligned}
f(x)-J f(x)= & \frac{1}{4096}\left(D f_{e}(-6 x, 2 x)+8 D f_{e}(-x, x)\right. \\
& \left.+56 D f_{e}(-2 x, x)+112 D f_{e}(-3 x, x)\right) \\
& +\frac{1}{512}\left(D f_{o}(-6 x, 2 x)+6 D f_{o}(-x, x)\right. \\
& \left.+42 D f_{o}(-2 x, x)+112 D f_{o}(-3 x, x)\right) .
\end{aligned}
$$

And, by (11) we obtain

$$
\begin{aligned}
&\|f(x)-J f(x)\| \\
& \leq \frac{1}{4096} ? D f_{e}(-6 x, 2 x)+8 D f_{e}(-x, x) \\
&+56 D f_{e}(-2 x, x)+112 D f_{e}(-3 x, x) ? \\
&+\frac{1}{512} ? D f_{o}(-6 x, 2 x)+6 D f_{o}(-x, x) \\
&+42 D f_{o}(-2 x, x)+112 D f_{o}(-3 x, x) ? \\
& \leq \frac{9 \varphi_{e}(-6 x, 2 x)+56 \varphi_{e}(-x, x)+392 \varphi_{e}(-2 x, x)+1008 \varphi_{e}(-3 x, x)}{4096} \\
&= \Phi(x),
\end{aligned}
$$

for every $x \in V$. It implies that $d(f, J f) \leq 1<\infty$ from the definition of $d$ and due to Proposition 1, the sequence $\left\{J^{n} f\right\}$ converges to only one fixed point $F: V \longrightarrow Y$ of $J$ in the set $T=\{g \in S: d(f, g)<\infty\}$ which implies (13). Moreover, by Proposition 1, we have

$$
d(f, F) \leq \frac{1}{1-L} d(f, J f) \leq \frac{1}{1-L},
$$

which implies (12).

Also, by the equality (8) in Lemma 2, since one has

$$
\begin{gathered}
\lim _{n \rightarrow \infty} \frac{1}{4096^{n}}\left\|\sum_{i=0}^{n}{ }_{n} C_{i}\left(\sum_{j=0}^{i}{ }_{i} C_{j}(-84)^{i-j} 1344^{j} D f_{e}\left(2^{3 n-i-j} x, 2^{3 n-i-j} y\right)\right)\right\| \\
\leq \lim _{n \rightarrow \infty} \frac{1}{4096^{n}} \sum_{i=0}^{n}{ }_{n} C_{i}\left(\sum_{j=0}^{i}{ }_{i} C_{j} 84^{i-j} 1344^{j} \varphi_{e}\left(2^{3 n-i-j} x, 2^{3 n-i-j} y\right)\right) \\
\leq \lim _{n \rightarrow \infty} \frac{1}{512^{n}} \sum_{i=0}^{n}{ }_{n} C_{i}\left(\sum_{j=0}^{i}{ }_{i} C_{j} 42^{i-j} 336^{j} \varphi_{e}\left(2^{3 n-i-j} x, 2^{3 n-i-j} y\right)\right)=0
\end{gathered}
$$

and

$$
\begin{gathered}
\lim _{n \rightarrow \infty} \frac{1}{512^{n}}\left\|\sum_{i=0}^{n}{ }_{n} C_{i}\left(\sum_{j=0}^{i}{ }_{i} C_{j}(-42)^{i-j} 336^{j} D f_{o}\left(2^{3 n-i-j} x, 2^{3 n-i-j} y\right)\right)\right\| \\
\leq \lim _{n \rightarrow \infty} \frac{1}{512^{n}} \sum_{i=0}^{n}{ }_{n} C_{i}\left(\sum_{j=0}^{i}{ }_{i} C_{j} 42^{i-j} 336^{j} \varphi_{e}\left(2^{3 n-i-j} x, 2^{3 n-i-j} y\right)\right)=0,
\end{gathered}
$$

for all $x, y \in V$, we obtain

$$
\begin{aligned}
D F(x, y)= & \lim _{n \rightarrow \infty}\left(\sum _ { i = 0 } ^ { n } { } _ { n } C _ { i } \sum _ { j = 0 } ^ { i } { } _ { i } C _ { j } \left(\frac{(-84)^{i-j} 1344^{j}}{4096^{n}} D f_{e}\left(2^{3 n-i-j} x\right)\right.\right. \\
& \left.\left.+\frac{(-42)^{i-j} 336^{j}}{512^{n}} D f_{o}\left(2^{3 n-i-j} x, 2^{3 n-i-j} y\right)\right)\right)=0,
\end{aligned}
$$

for every $x, y \in V$.

Therefore, $F$ is the unique solution of the functional equation (4) with (12). Finally, we see that if $F$ is a solution of the sextic functional equation (4) with $F(0)=0$, then we can derive that $F$ is a fixed point of $J$ from the equality

$$
\begin{aligned}
F(x)-J F(x)= & \frac{1}{4096}\left(D F_{e}(-6 x, 2 x)+8 D F_{e}(-x, x)\right. \\
& \left.+56 D F_{e}(-2 x, x)+112 D F_{e}(-3 x, x)\right) \frac{1}{512} \\
& \cdot\left(D F_{o}(-6 x, 2 x)+6 D F_{o}(-x, x)+42 D F_{o}\right. \\
& \left.\cdot(-2 x, x)+112 D F_{o}(-3 x, x)\right) .
\end{aligned}
$$

In next corollary, we will consider special function $\varphi(x$, $y)=\|x\|^{p}+\|y\|^{p}$ in Theorem 3 to compare with the results in [31]. 
Corollary 4. Let $X$ be a real normed space, $\theta$ be as in Lemma 2 , and $p$ be a fixed real number such that $0<p<\log _{2}(4 \sqrt{21}$ $-14)$. If $f: X \longrightarrow Y$ satisfies the equality $f(0)=0$ and the inequality

$$
\|D f(x, y)\| \leq\|x\|^{p}+\|y\|^{p}
$$

for all $x, y \in X$, then there exists a unique solution mapping $F: V \longrightarrow Y$ of (4) satisfying the inequality

$$
\|f(x)-F(x)\| \leq \frac{\left(1512+401 \cdot 2^{p}+1008 \cdot 3^{p}+9 \cdot 6^{p}\right)(2 \sqrt{21}-7)\|x\|^{p}}{8192\left(4 \sqrt{21}-14-2^{p}\right)},
$$

for all $x \in X$.

Proof. If we put $\varphi(x, y)=\|x\|^{p}+\|y\|^{p}$ and $L=2^{p} / 4 \sqrt{21}-14$, then we have the equalities $\varphi(2 x, 2 y)=2^{p} \varphi(x, y)=(4 \sqrt{21}$ 14) $L \varphi(x, y)$ for all $x, y \in X$. So the condition (6) in Lemma 2 holds for all $x, y \in X$. According to Theorem 3, there exists a unique solution mapping $F: V \longrightarrow Y$ of (4) satisfying the inequality (16) for all $x, y \in X$.

Next, we will try to prove the stability of the sextic functional equation (4) from another point of view. For that, we first will introduce useful facts in the following lemma.

Lemma 5. Let $\theta$ be a real constant such that $0<\theta<\pi / 4$ and $\cos (3 \theta)=637 / 77 \sqrt{77}$. Let $\varphi: V^{2} \longrightarrow[0, \infty)$ be a function for which there exists a constant $0<L<1$ such that

$$
L \varphi(2 x, 2 y) \geq(8 \sqrt{77} \cos \theta+28) \varphi(x, y)
$$
97.8 ,

for all $x, y \in V$. Then, we have $97<8 \sqrt{77} \cos \theta+28<$ $\frac{84}{(8 \sqrt{77} \cos \theta+28)}+\frac{1344}{(8 \sqrt{77} \cos \theta+28)^{2}}+\frac{4096}{(8 \sqrt{77} \cos \theta+28)^{3}}=1$,

and the equality

$$
\lim _{n \rightarrow \infty} \sum_{i=0}^{n}{ }_{n} C_{i} \sum_{j=0}^{i}{ }_{i} C_{j} 84^{j} 1344^{i-j} 4096^{n-i} \varphi_{e}\left(\frac{x}{2^{3 n-i-j}}, \frac{y}{2^{3 n-i-j}}\right)=0,
$$

holds for all $x, y \in V$.

Proof. When $0<\theta<\pi / 4$ and $\cos (3 \theta)=637 / 77 \sqrt{77}$, it is not difficult to see that $0<3 \theta<0.33997$ and $97<8 \sqrt{77} \cos \theta+$ $28<97.8$ in the trigonometric function table. Also, we obtain the equality (18) from the following calculation:

$$
\begin{aligned}
4096 & +1344(8 \sqrt{77} \cos \theta+28)+84(8 \sqrt{77} \cos \theta+28)^{2} \\
& -(8 \sqrt{77} \cos \theta+28)^{3} \\
= & 4096+10752 \sqrt{77} \cos \theta+37632+413952 \cos ^{2} \theta \\
& +37632 \sqrt{77} \cos \theta+65856-39424 \sqrt{77} \cos ^{3} \theta \\
& -413952 \cos ^{2} \theta-18816 \sqrt{77} \cos \theta-21952 \\
= & -9856 \sqrt{77}\left(4 \cos ^{3} \theta-3 \cos \theta\right)+81536 \\
= & -9856 \sqrt{77} \cos (3 \theta)+81536=0 .
\end{aligned}
$$

And by (17) and (18), we have

$$
\begin{aligned}
& \sum_{i=0}^{n}{ }_{n} C_{i} \sum_{j=0}^{i}{ }_{i} C_{j} 84^{j} 1344^{i-j} 4096^{n-i} \varphi_{e}\left(\frac{x}{2^{3 n-i-j}}, \frac{y}{2^{3 n-i-j}}\right) \\
& \leq \sum_{i=0}^{n}{ }_{n} C_{i} \sum_{j=0}^{i}{ }_{i} C_{j} 84^{j}\left(\frac{1344 L}{8 \sqrt{77} \cos \theta+28}\right)^{i-j} 4096^{n-i} \varphi_{e} \\
& \quad \times\left(\frac{x}{2^{3 n-2 i}}, \frac{y}{2^{3 n-2 i}}\right) \\
&=\sum_{i=0}^{n}{ }_{n} C_{i}\left(84+\frac{1344 L}{8 \sqrt{77} \cos \theta+28}\right)^{i} 4096^{n-i} \varphi_{e}\left(\frac{x}{2^{3 n-2 i}}, \frac{y}{2^{3 n-2 i}}\right) \\
& \leq \sum_{i=0}^{n}{ }_{n} C_{i}\left(84+\frac{1344 L}{8 \sqrt{77} \cos \theta+28}\right)^{i}\left(\frac{4096 L^{2}}{(8 \sqrt{77} \cos \theta+28)^{2}}\right)^{n-i} \\
& \times \varphi_{e}\left(\frac{x}{2^{n}}, \frac{y}{2^{n}}\right) \\
& \leq\left(84+\frac{1344 L}{8 \sqrt{77} \cos \theta+28}+\frac{4096 L^{2}}{(8 \sqrt{77} \cos \theta+28)^{2}}\right)^{n} \\
& \times\left(\frac{L}{8 \sqrt{77} \cos \theta+28}\right)^{n} \varphi_{e}(x, y) \\
& \leq\left(\frac{84}{8 \sqrt{77} \cos \theta+28}+\frac{1344}{(8 \sqrt{77} \cos \theta+28)^{2}}\right. \\
&\left.+\frac{4096}{(8 \sqrt{77} \cos \theta+28)^{3}}\right)^{n} L^{n} \varphi_{e}(x, y)=L^{n} \varphi_{e}(x, y),
\end{aligned}
$$

for all $x, y \in V$. Therefore, by taking the limit, we complete the proof of (19).

In the following theorem, we will prove the stability of the solution for the sextic functional equation (4) with different types of functions compared to Theorem 3 .

Theorem 6. Let $\theta, L$, and $\varphi$ be as in Lemma 5. If $f: V \longrightarrow Y$ is a mapping such that the inequality (11) in Theorem 3 holds for every $x, y \in V$, then there exists only one solution $F: V$ $\longrightarrow Y$ of (4) satisfying the following inequality 


$$
\|f(x)-F(x)\| \leq \frac{\Psi(x)}{1-L}
$$

for every $x \in V$, where

$$
\begin{aligned}
\Psi(x)= & 2 \varphi_{e}\left(\frac{-3 x}{4}, \frac{x}{4}\right)+14 \varphi_{e}\left(\frac{-x}{8}, \frac{x}{8}\right)+98 \varphi_{e}\left(\frac{-x}{4}, \frac{x}{8}\right) \\
& +224 \varphi_{e}\left(\frac{-3 x}{8}, \frac{x}{8}\right) .
\end{aligned}
$$

In particular, $F$ is represented by

$$
\begin{aligned}
F(x)= & \lim _{n \rightarrow \infty} \sum_{i=0}^{n}{ }_{n} C_{i} \sum_{j=0}^{i}{ }_{i} C_{j}\left[42^{j}(-336)^{i-j} 512^{n-i} f_{o}\left(\frac{x}{2^{3 n-i-j}}\right)\right. \\
& \left.+84^{j}(-1344)^{i-j} 4096^{n-i} f_{e}\left(\frac{x}{2^{3 n-i-j}}\right)\right],
\end{aligned}
$$

for any $x \in V$.

Proof. Similar to Theorem 3, we consider the set $S$ which contains all functions $g: V \longrightarrow Y$ with $g(0)=0$, and we define a generalized metric on $S$ as

$d(g, h)=\inf \left\{K \in R_{+} \mid\|g(x)-h(x)\| \leq K \Psi(x)\right.$ for all $\left.x \in V\right\}$.

We now consider the mapping $J: S \longrightarrow S$ defined by

$$
\begin{aligned}
J g(x)= & 63 g\left(\frac{x}{2}\right)+21 g\left(-\frac{x}{2}\right)-840 g\left(\frac{x}{4}\right)-504 g\left(-\frac{x}{4}\right) \\
& +2304 g\left(\frac{x}{8}\right)+1792 g\left(-\frac{x}{8}\right),
\end{aligned}
$$

for every $x \in V$. Then, similar to Theorem 3, by mathematical induction, we obtain that

$$
\begin{aligned}
J^{n} g(x)= & \sum_{i=0}^{n}{ }_{n} \mathrm{C}_{i} \sum_{j=0}^{i}{ }_{i} \mathrm{C}_{j}\left(42^{j}(-336)^{i-j} 512^{n-i} g_{o}\left(\frac{x}{2^{3 n-i-j}}\right) 33 m m\right. \\
& \left.+84^{j}(-1344)^{i-j} 4096^{n-i} g_{e}\left(\frac{x}{2^{3 n-i-j}}\right)\right)
\end{aligned}
$$

holds for every $n \in \mathbb{N}$ and $x \in V$.

Let $g, h \in S$ and we assume $K \in[0, \infty]$ as an arbitrary constant with $d(g, h) \leq K$. By the definition of $d$ and (18) in Lemma 5, we have

$$
\begin{aligned}
& \|J g(x)-J h(x)\| \\
& \leq 63\left\|g\left(\frac{x}{2}\right)-h\left(\frac{x}{2}\right)\right\|+21\left\|g\left(-\frac{x}{2}\right)-h\left(-\frac{x}{2}\right)\right\| \\
& \quad+840\left\|g\left(\frac{x}{4}\right)-h\left(\frac{x}{4}\right)\right\|+504\left\|g\left(-\frac{x}{4}\right)-h\left(-\frac{x}{4}\right)\right\| \\
& \quad+2304\left\|g\left(\frac{x}{8}\right)-h\left(\frac{x}{8}\right)\right\|+1792\left\|g\left(-\frac{x}{8}\right)-h\left(-\frac{x}{8}\right)\right\|
\end{aligned}
$$

$$
\begin{aligned}
\leq & 84 K \Psi\left(\frac{x}{2}\right)+1344 K \Psi\left(\frac{x}{4}\right)+4096 K \Psi\left(\frac{x}{8}\right) \\
\leq & \frac{84 L K \Psi(x)}{8 \sqrt{77} \cos \theta+28}+\frac{1344 L^{2} K \Psi(x)}{(8 \sqrt{77} \cos \theta+28)^{2}} \\
& +\frac{4096 L^{3} K \Psi(x)}{(8 \sqrt{77} \cos \theta+28)^{3}} \\
\leq & \left(\frac{84}{8 \sqrt{77} \cos \theta+28}+\frac{1344}{(8 \sqrt{77} \cos \theta+28)^{2}}\right. \\
& \left.+\frac{4096}{(8 \sqrt{77} \cos \theta+28)^{3}}\right) L K \Psi(x) \\
\leq & L K \Psi(x),
\end{aligned}
$$

for every $x \in V$, which implies that

$$
d(J g, J h) \leq L d(g, h)
$$

for all $g, h \in S$, where $0<L<1$. So, with the Lipschitz constant $L, J$ is a strictly contractive self-mapping of $S$, where $0<L<1$.

Moreover, by the definition of $D f(x, y)$, with long and tedious calculation, we have

$$
\begin{aligned}
f(x)-J f(x)= & {\left[D f_{e}\left(\frac{-3 x}{4}, \frac{x}{4}\right)+8 D f_{e}\left(\frac{-x}{8}, \frac{x}{8}\right)+56 D f_{e}\right.} \\
& \left.\cdot\left(\frac{-x}{4}, \frac{x}{8}\right)+112 D f_{e}\left(\frac{-3 x}{8}, \frac{x}{8}\right)\right] \\
& +\left[D f_{o}\left(\frac{-3 x}{4}, \frac{x}{4}\right)+6 D f_{o}\left(\frac{-x}{8}, \frac{x}{8}\right)+42 D f_{o}\right. \\
& \left.\cdot\left(\frac{-x}{4}, \frac{x}{8}\right)+112 D f_{o}\left(\frac{-3 x}{8}, \frac{x}{8}\right)\right] .
\end{aligned}
$$

And, by (11) in assumption, we obtain

$$
\begin{aligned}
\|f(x)-J f(x)\| \leq & \| D f_{e}\left(\frac{-3 x}{4}, \frac{x}{4}\right)+8 D f_{e}\left(\frac{-x}{8}, \frac{x}{8}\right) \\
& +56 D f_{e}\left(\frac{-x}{4}, \frac{x}{8}\right)+112 D f_{e}\left(\frac{-3 x}{8}, \frac{x}{8}\right) \| \\
& +\| D f_{o}\left(\frac{-3 x}{4}, \frac{x}{4}\right)+6 D f_{o}\left(\frac{-x}{8}, \frac{x}{8}\right) \\
& +42 D f_{o}\left(\frac{-x}{4}, \frac{x}{8}\right)+112 D f_{o}\left(\frac{-3 x}{8}, \frac{x}{8}\right) \|
\end{aligned}
$$




$$
\begin{array}{r}
\leq 2 \varphi_{e}\left(\frac{-3 x}{4}, \frac{x}{4}\right)+14 \varphi_{e}\left(\frac{-x}{8}, \frac{x}{8}\right)+98 \varphi_{e} \\
\cdot\left(\frac{-x}{4}, \frac{x}{8}\right)+224 \varphi_{e}\left(\frac{-3 x}{8}, \frac{x}{8}\right)=\Psi(x),
\end{array}
$$

for every $x \in V$.

It implies that $d(f, J f) \leq 1<\infty$ by the definition of $d$. Therefore, according to Proposition 1, the sequence $\left\{J^{n} f\right\}$ converges to only one fixed point $F: V \longrightarrow Y$ of $J$ in the set $T=\{g \in S \mid d(f, g)<\infty\}$, which is represented by (23) for every $x \in V$.

We also due to Proposition 1 obtain that

$$
d(f, F) \leq \frac{1}{1-L} d(f, J f) \leq \frac{1}{1-L}
$$

which implies (22).

Now, by (19) in Lemma 5, since we have

$$
\begin{aligned}
& \lim _{n \rightarrow \infty}\left\|\sum_{i=0}^{n}{ }_{n} C_{i} \sum_{j=0}^{i}{ }_{i} C_{j} 84^{j}(-1344)^{i-j} 4096^{n-i} D f_{e}\left(\frac{x}{2^{3 n-i-j}}, \frac{y}{2^{3 n-i-j}}\right)\right\| \\
& \leq \lim _{n \rightarrow \infty} \sum_{i=0}^{n}{ }_{n} C_{i} \sum_{j=0}^{i}{ }_{i} C_{j} 84^{j} 1344^{i-j} 4096^{n-i} \varphi_{e}\left(\frac{x}{2^{3 n-i-j}}, \frac{y}{2^{3 n-i-j}}\right)=0, \\
& \lim _{n \rightarrow \infty}\left\|\sum_{i=0}^{n}{ }_{n} C_{i} \sum_{j=0}^{i}{ }_{i} C_{j} 42^{j}(-336)^{i-j} 512^{n-i} D f_{o}\left(\frac{x}{2^{3 n-i-j}}, \frac{y}{2^{3 n-i-j}}\right)\right\| \\
& \leq \lim _{n \rightarrow \infty} \sum_{i=0}^{n}{ }_{n} C_{i} \sum_{j=0}^{i}{ }_{i} C_{j} 42^{j} 336^{i-j} 512^{n-i} \varphi_{e}\left(\frac{x}{2^{3 n-i-j}}, \frac{y}{2^{3 n-i-j}}\right) \\
& \leq \lim _{n \rightarrow \infty} \sum_{i=0}^{n}{ }_{n} C_{i} \sum_{j=0}^{i}{ }_{i} C_{j} 84^{j} 1344^{i-j} 4096^{n-i} \varphi_{e}\left(\frac{x}{2^{3 n-i-j}}, \frac{y}{2^{3 n-i-j}}\right)=0,
\end{aligned}
$$

for all $x, y \in V$, due to the equality (23), we obtain

$$
\begin{aligned}
D F(x, y)= & \lim _{n \rightarrow \infty} \sum_{i=0}^{n}{ }_{n} C_{i} \sum_{j=0}^{i}{ }_{i} C_{j} 42^{j}(-336)^{i-j} 512^{n-i} D f_{o} \\
& \cdot\left(\frac{x}{2^{3 n-i-j}}, \frac{y}{2^{3 n-i-j}}\right)+\lim _{n \rightarrow \infty} \sum_{i=0}^{n}{ }_{n} C_{i} \sum_{j=0}^{i}{ }_{i} C_{j} 84^{j} \\
& \cdot(-1344)^{i-j} 4096^{n-i} D f_{e}\left(\frac{x}{2^{3 n-i-j}}, \frac{y}{2^{3 n-i-j}}\right)=0,
\end{aligned}
$$

which conclude that $F$ is a solution of the sextic functional equation (4).

Finally, we see that if $F$ is a solution of the sextic functional equation (4), then the equality

$$
\begin{aligned}
F(x)-J F(x)= & {\left[D F_{e}\left(\frac{-3 x}{4}, \frac{x}{4}\right)+8 D F_{e}\left(\frac{-x}{8}, \frac{x}{8}\right)\right.} \\
& \left.+56 D F_{e}\left(\frac{-x}{4}, \frac{x}{8}\right)+112 D F_{e}\left(\frac{-3 x}{8}, \frac{x}{8}\right)\right]
\end{aligned}
$$

$$
\begin{aligned}
& +\left[D F_{o}\left(\frac{-3 x}{4}, \frac{x}{4}\right)+6 D F_{o}\left(\frac{-x}{8}, \frac{x}{8}\right)\right. \\
& \left.+42 D F_{o}\left(\frac{-x}{4}, \frac{x}{8}\right)+112 D F_{o}\left(\frac{-3 x}{8}, \frac{x}{8}\right)\right],
\end{aligned}
$$

implies that $F$ is a fixed point of $J$.

In next corollary, we will consider special function $\varphi(x$, $y)=\|x\|^{p}+\|y\|^{p}$ in Theorem 6 to compare with the results in [31].

Corollary 7. Let $X$ be a real normed space, $\theta$ be as in Lemma 5 , and $p$ be a fixed real number such that $\log _{2}(8 \sqrt{77} \cos \theta+$ $28)<p$. If $f: X \longrightarrow Y$ satisfies the equality $f(0)=0$ and the inequality (15) for all $x, y \in X$, then there exists a unique solution mapping $F: V \longrightarrow Y$ of (4) such that

$$
\|f(x)-F(x)\| \leq \frac{\left(175+50 \cdot 2^{p}+112 \cdot 3^{p}+6^{p}\right)\|x\|^{p}}{4^{p}\left(2^{p}-8 \sqrt{77} \cos \theta+28\right)}
$$

for all $x \in X$.

Proof. If we put $\varphi(x, y)=\|x\|^{p}+\|y\|^{p}$ and $L=8 \sqrt{77} \cos \theta+$ $28 / 2^{p}$, then we have the equalities $L \varphi(2 x, 2 y)=2^{p} L \varphi(x, y)=$ $(8 \sqrt{77} \cos \theta+28) \varphi(x, y)$ for all $x, y \in X$. So the condition (17) in Lemma 5 holds for all $x, y \in X$. According to Theorem 6, there exists a unique solution mapping $F: V \longrightarrow Y$ of (4) satisfying the inequality (24) for all $x, y \in X$.

\section{Data Availability}

No data are available for this study.

\section{Conflicts of Interest}

The authors declare that there are no conflicts of interest regarding the publication of this article.

\section{Acknowledgments}

This work was supported by Hallym University Research Fund (HRF-201910-014).

\section{References}

[1] S. M. Ulam, A Collection of Mathematical Problems, Interscience, New York, NY, USA, 1960.

[2] D. H. Hyers, "On the stability of the linear functional equation," Proceedings of the National Academy of Sciences of the United States of America, vol. 27, no. 4, pp. 222-224, 1941.

[3] P. Găvruta, "A generalization of the Hyers-Ulam-Rassias stability of approximately additive mappings," Journal of Mathematical Analysis and Applications, vol. 184, no. 3, pp. 431-436, 1994.

[4] S.-M. Jung, "On the Hyers-Ulam stability of the functional equations that have the quadratic property," Journal of 
Mathematical Analysis and Applications, vol. 222, no. 1, pp. 126-137, 1998.

[5] B. Khosravi, M. B. Moghimi, and A. Najati, "Generalized Hyers-Ulam stability of a functional equation of Hosszu type," Nonlinear Functional Analysis and Applications, vol. 23, no. 1, pp. 157-166, 2018.

[6] Y.-H. Lee and K.-W. Jun, "On the stability of approximately additive mappings," Proceedings of the American Mathematical Society, vol. 128, pp. 1361-1369, 2000.

[7] Y.-H. Lee and S.-M. Jung, "A general theorem on the fuzzy stability of a class of functional equations including quadraticadditive functional equations," Nonlinear Functional Analysis and Applications, vol. 23, no. 2, pp. 353-368, 2018.

[8] Y.-H. Lee and S.-M. Jung, "Stability of some cubic-additive functional equations," Nonlinear Functional Analysis and Applications, vol. 25, no. 1, pp. 35-54, 2020.

[9] S. Ostadbashi and M. Soleimaninia, "On the stability of the orthogonal pexiderized quartic functional equations," Nonlinear Functional Analysis and Applications, vol. 20, no. 4, pp. 539-549, 2015.

[10] T. M. Rassias, "On the stability of the linear mapping in Banach spaces," Proceedings of the American Mathematical Society, vol. 72, no. 2, pp. 297-300, 1978.

[11] S. Alshybani, S. M. Vaezpour, and R. Saadati, "Generalized Hyers-Ulam stability of sextic functional equation in random normed spaces," Journal of Computational Analysis and Applications, vol. 24, no. 2, pp. 370-381, 2018.

[12] S. Alshybani, S. M. Vaezpour, and R. Saadati, "Stability of the sextic functional equation in various spaces," Journal of Inequalities and Special Functions, vol. 9, no. 4, pp. 8-27, 2018.

[13] Y. J. Cho, M. B. Ghaemi, M. Choubin, and M. E. Gordji, "On the Hyers-Ulam stability of sextic functional equations in $\beta$ -homogeneous probabilistic modular spaces," Mathematical Inequalities \& Applications, vol. 16, no. 4, pp. 1097-1114, 2013.

[14] I. I. EL-Fassi, "New stability results for the radical sextic functional equation related to quadratic mappings in $(2, \beta)$ -Banach spaces," Journal of Fixed Point Theory and Applications, vol. 20, pp. 1-17, 2018.

[15] K. Ravi and S. Sabarinathan, "Generalized Hyers-Ulam stability of a sextic functional equation in paranormed spaces," International Journal of Mathematics Trends and Technology, vol. 9, no. 1, pp. 61-69, 2014.

[16] T. Xu, J. Rassias, M. Rassias, and W. Xu, "A fixed point approach to the stability of quintic and sextic functional equations in quasi-normed spaces," Journal of Inequalities and Applications, vol. 2010, no. 1, Article ID 423231, 2010.

[17] T. Xu, J. Rassias, M. Rassias, and W. Xu, "Stability of quintic and sextic functional equations in non-archimedean fuzzy normed spaces," in 2011 Eighth International Conference on Fuzzy Systems and Knowledge Discovery (FSKD), Shanghai, China, July 2011.

[18] M. Almahalebi, A. Charifi, and S. Kabbaj, "Hyperstability of a monomial functional equation," Journal of Scientific Research and Reports, vol. 3, no. 20, pp. 2685-2693, 2014.

[19] M. Almahalebi, M. Sirouni, A. Charifi, and S. Kabbaj, "Fuzzy stability of the monomial functional equation," Journal Nonlinear Analysis and Application, vol. 2014, pp. 1-13, 2014.

[20] L. Cădariu and V. Radu, "A general fixed point method for the stability of the monomial functional equation," Carpathian Journal of Mathematics, vol. 28, no. 1, pp. 25-36, 2012.
[21] Z. Kaiser, "On stability of the monomial functional equation in normed spaces over fields with valuation," Journal of Mathematical Analysis and Applications, vol. 322, no. 2, pp. 11881198, 2006.

[22] Y.-H. Lee, "On the stability of the monomial functional equation," Bulletin of the Korean Mathematical Society, vol. 45, no. 2, pp. 397-403, 2008.

[23] Y.-H. Lee, "Stability of a monomial functional equation on a restricted domain," Mathematics, vol. 5, no. 4, p. 53, 2017.

[24] A. K. Mirmostafaee, "Non-Archimedean stability of the monomial functional equations," Tamsui Oxford Journal of Mathematical Sciences, vol. 26, no. 2, pp. 221-235, 2010.

[25] A. K. Mirmostafaee, "Stability of the monomial functional equation in quasi normed spaces," Bulletin of the Korean Mathematical Society, vol. 47, no. 4, pp. 777-785, 2010.

[26] C. Zaharia, "On the probabilistic stability of the monomial functional equation," Journal of Nonlinear Sciences and Applications, vol. 6, no. 1, pp. 51-59, 2013.

[27] J. Baker, "A general functional equation and its stability," Proceedings of the American Mathematical Society, vol. 133, no. 6, pp. 1657-1664, 2005.

[28] K.-W. Jun and H.-M. Kim, "On the Hyers-Ulam-Rassias stability of a general cubic functional equation," Mathematical Inequalities \& Applications, vol. 6, no. 2, pp. 289-302, 2003.

[29] Y.-H. Lee, "On the generalized Hyers-Ulam stability of the generalized polynomial function of degree 3," Tamsui Oxford Journal of Mathematical Sciences, vol. 24, no. 4, pp. 429-444, 2008.

[30] Y.-H. Lee, "On the Hyers-Ulam-Rassias stability of a general quartic functional equation," East Asian Mathematical Journal, vol. 35, pp. 351-356, 2019.

[31] Y.-H. Lee, "On the Hyers-Ulam-Rassias stability of a general quintic functional equation and a general sextic functional equation," Mathematics, vol. 7, no. 6, p. 510, 2019.

[32] L. Cădariu and V. Radu, "Fixed points and the stability of Jensen's functional equation," Journal ofInequalities in Pure and Applied Mathematics, vol. 4, no. 1, pp. 1-15, 2003.

[33] L. Cădariu and V. Radu, "Fixed points and the stability of quadratic functional equations," Analele Universitatii de Vest din Timisoara, vol. 41, pp. 25-48, 2003.

[34] L. Cădariu and V. Radu, "On the stability of the Cauchy functional equation: a fixed point approach in Iteration Theory," Grazer Mathematische Berichte, vol. 346, pp. 43-52, 2004.

[35] N. Saleem, J. Vujakovic, W. U. Baloch, and S. Radenovic, "Coincidence point results for multivalued suzuki type mappings using $\theta$-contraction in b-metric spaces," Mathematics, vol. 7, no. 11, article 1017, 2019.

[36] J. B. Diaz and B. Margolis, "A fixed point theorem of the alternative for contractions on a generalized complete metric space," Bulletin of the American Mathematical Society, vol. 74, no. 2, pp. 305-310, 1968.

[37] S.-M. Jung and T.-S. Kim, "A fixed point approach to the stability of the cubic functional equation," Boletín de la Sociedad Matemática Mexicana, vol. 12, no. 1, pp. 51-57, 2006. 